\title{
Fundamentos de la Enseñanza por Competencias a Nivel de Postgrado en dos Universidades Públicas Chilenas
}

\author{
Miguel A. Bustamante ${ }^{(1) \star}$, Claudio H. Oyarzún ${ }^{(2)}$, Maderline L. Grandón (3) y Caroline G. Abarza (1) \\ (1) Escuela de Ingeniería Comercial, Facultad de Economía y Negocios, Universidad de Talca Campus \\ Lircay. Avenida Lircay S/N, Talca-Chile (e-mail: mabu@utalca.cl) \\ (2) Instituto de Investigación y Desarrollo Educacional, IIDE, Universidad de Talca Campus Lircay. \\ Avenida Lircay S/N, Talca-Chile (e-mail: cloyarzun@utalca.cl) \\ (3) Centro de Gestión en Instituciones de Salud, CEGIS, Universidad de Talca Campus Lircay. Avenida \\ Lircay S/N, Talca-Chile (e-mail: mgrandon@utalca.cl) \\ * autor a quien debe ser dirigida la correspondencia
}

Recibido Mar. 12, 2015; Aceptado May. 15, 2015; Versión final Jun. 10, 2015, Publicado Dic. 2015

\begin{abstract}
Resumen
Se identifican los fundamentos de la práctica docente de postgrado en el contexto de la enseñanza basada en competencias en dos universidades públicas chilenas. El estudio, cualitativo, no experimental y exploratorio, se realizó mediante el análisis de las actividades académicas de profesores de diversos perfiles. Los datos fueron procesados mediante el análisis de contenido de los discursos escritos recogidos de una muestra de 66 profesores. Entre los principales resultados se encuentra una dicotomía entre declaración y práctica docente que se enfoca en el modelo tradicional de enseñanza, de carácter cognitivo, más que en el desarrollo de capacidades asociadas a la enseñanza basada en competencias. En una de las universidades se evidencian concepciones ambivalentes que los sitúan en el modelo de enseñanza tradicional, por objetivo. En la otra se observa un perfil docente enfocado en el profesionalismo.
\end{abstract}

\section{Foundations of the Teaching by Competence at level of Graduate degree in two Chilean Public Universities}

\begin{abstract}
The foundations of the educational practice of graduate studies are identified in the context of the teaching by competence in two Chilean public universities. The study, qualitative, not experimental and exploratory, was carried out by means of the analysis of the academic activities of professors of diverse profiles. The data were processed by means of the analysis of content of the written speeches collected from a sample of 66 professors. Among the main results a dichotomy is found between declaration and educational practice that is focused in the traditional pattern of teaching, of cognitive character, more than in the development of capacities associated to the teaching by competence. In one of the universities, ambivalent conceptions are evidenced that locate them in the pattern of traditional teaching, by objective. In the other one, an educational profile focused on professionalism is observed.
\end{abstract}

Keywords: teaching and training, professional training, pedagogical practice, teaching method, competences 


\section{INTRODUCCIÓN}

Transcurrida más de una década de definida la educación terciaria en Chile (Jiménez et al., 2013), de haberse incorporado progresivamente los movimientos mundiales de armonización y homologación (Didou, 1996) y de permear la enseñanza con el enfoque de competencias profesionales y transversales(Rodríguez et al., 2011; Siqueira, 2012), el presente trabajo analiza la práctica docente a nivel de postgrado, con el propósito de colaborar a la inteligibilidad de los fundamentos de la práctica docente conforme al paradigma de la enseñanza basada en competencias (Cardoso et al., 2013).

Asumiendo en este trabajo que las innovaciones del método docente contiene una dinámica constructivista compleja en materia didáctica y procedimental para la formación de personas adultas insertas en estudios universitarios, como son los niveles de postgrado (Castaño y Garín, 2012; Davini, 2008; García et al., 2008) y reconociendo que muchos docentes, para lograr eficacia en su enseñanza, han adoptado estrategias y técnicas que se asocian a la enseñanza magistral (Camargo y Pardo, 2008), la innovación asociada a enseñanza con enfoque en competencias, ha dado pie a la revalorización de la capacidad de los docentes para detectar gestos, preguntas y receptividad de los estudiantes en un contexto de modernización de la docencia de postgrado (Palominos et al., 2014; Litwin, 2008).

Por otra parte, reconociendo que el sistema educativo formal es, en general, relativamente estable e impermeable a los cambios (Pedraja, 2012; Muñoz, 2012), la discusión que se plantea en este trabajo puede contribuir a la literatura de la enseñanza de nivel superior recogiendo por un lado ventajas y desventajas (Monereo et al., 2000) que han influido en la conducta de docentes de diversos perfiles profesionales (MarínGarcía y Conchado-Peiró, 2012) y por otra, relevando prácticas que conllevan una secuencia temporal y lógica centrada en la eficacia. Para unos implica derivaciones complejas de tipo "Sicológicas, Lógicas y Contextuales" de adecuación al contexto por parte de estudiante insertos en sus propias realidades laborales (De Miguel, 2005)y para otros, la adopción de un método de enseñanza por competencia, a nivel de postgrado, que supera su definición etimológica "metha" y "odos" - como un "camino" al más "allá"-dado por objetivos, recursos y procedimientos diferenciales que se adicionan al proceso de enseñanza-aprendizaje (Palominos et al., 2014; Monereo et al., 2000), para el desarrollo de capacidades, habilidades y, finalmente, competencias profesionales de postgrado.

El enfoque de Educación Basado en Competencias, asume que la persona competente es capaz de dar respuesta a los problemas que enfrenta (Zabalza, 2007); aprende a movilizar e integrar actitudes y procedimientos para resolver "situaciones problema" movilizando referentes científicos, culturales, actitudinales interrelacionados (Barbier, 1999). Son estas movilizaciones las que, progresivamente, modifican y mejoran el aprendizaje (De Miguel, 2005) generando un "alineamiento constructivo" (Biggs, 2005 citado por De Miguel, 2005) que ordena objetivos, métodos y hasta sistemas de evaluación, donde el centro de atención está en la didáctica de co-construcción que rompe con la línea tradicional de profesor, contenido, método y evaluación (Perrenoud, 2001).

Desde la perspectiva del perfeccionamiento docente (Palominos et al., 2014) el análisis de actividades que sugiere este trabajo incorpora una nueva vertiente de aprendizaje que da sentido y propósito a la inteligibilidad que cada persona puede lograr respecto de sí mismo (Barbier, 1999). Por una parte, reflexionando las propias vivencias en singularidad y, por otra, haciendo cambios a prácticas habituales que generan homeostasis en contextos de cambio (Barbier, 2004; Barbier, 1999). En lo esencial el dominio de una competencia, está relacionado con el pensamiento, reflexivo, lógico, analítico, además del liderazgo y la capacidad de resolución de problemas, donde el individuo es capaz de intencionar, junto al pensamiento práctico, sus capacidades para seleccionar el curso de acción más apropiado a una determinada realidad (Gagné, 1971; Barbier y Galatanu, 2000).

La "enseñanza por competencias... en el contexto de la formación profesional", pone de manifiesto que los docentes aceptan la inminencia de la diversidad de sus estudiantes (Díaz, et al., 2010) que deben canalizar el pensamiento deliberativo, la toma de decisiones y el examen de puntos de vista de sus estudiantes antes de emitir un juicio o ejecutar una acción sobre ellos (García et al., 2008). Además, otros autores como Camargo y Pardo (2008), definen la enseñanza basada en competencias en términos de la guía, colaboración y ayuda del docente para la aprehensión de contenidos y procedimientos a través de la "interpretación de eventos significativos, que develan estructuras de conciencia de la acción..." (Patiño y Rojas, 2009) en beneficio del estudiante.

Conforme lo indicado, el presente trabajo determina los fundamentos de la práctica docente de postgrado de dos universidades, identificadas como Universidad "A" y Universidad "B" frente a la implementación del currículum basado en competencias, recogiendo lo que se ha dado en denominar "contenido manifiesto" como dato real y "el contenido latente" como aquel que debe ser inferido (Krippendorff, 1990) para comprender la realidad. 


\section{METODOLOGÍA}

El trabajo de campo recogió las descripciones de los hechos tal como son relatados en el contexto en el que se producen (Cook y Reichardt, 1997) dando pie a un estudio no experimental, ex post facto utilizando el método de "análisis de actividades" (Bustamante et al., 2009) como medio de acercamiento al quehacer académico de profesores de diversos perfiles e insertos en sus respectivas realidades (Marín-García y Conchado-Peiró, 2012).

Se recogieron datos primarios por medio de entrevistas de explicitación escritas en un texto espontaneo de inteligibilidad de las actividades docentes y se distinguieron las verbalizaciones del relato y las descripciones del desempeño docente. El procesamiento se realizó mediante el análisis de contenido (Krippendorff, 1990; Ruiz, 2009) identificando elementos representativos tales como letras, sílabas, lexemas, fonemas, palabras, frases y, comentarios abiertos (Moreno et al., 2014, Glaser y Strauss, 1999), entre otras, para su clasificación bajo la forma de variables y categorías que explican el fenómeno investigado, evitando verlos e interpretarlos como si fuesen un conjunto azaroso de escritos dispersos (Krippendorff, 1990).

Para garantizar la calidad de los datos, el muestreo fue de tipo no probabilístico seleccionando profesores de postgrado de acuerdo con criterios estratégicos tales como conocimientos de la situación, facilidad, voluntariedad, etc. (Abela, 2002). El criterio de inclusión fue de docentes de categoría de planta de las dos Universidades en proceso de innovación curricular. Mediante la fórmula (1) se elaboró un listado de las prácticas recurrentes que conforman categorías de significado. En esta fórmula la expresión $X_{i}$ corresponde al valor de la variable $i$ y $n$ al número de variables.

$$
\sum X i / n
$$

Además, para comparar dichas palabras con las restantes dentro del sistema en análisis, se procedió a la estandarización de las frecuencias en torno a la media descontando el efecto de la dispersión de los datos a través de la fórmula (2).

$$
X=(X i-\mu) / \sigma
$$

La expresión $X$ es el valor de la variable estandarizada, $X_{i}$ corresponde al valor de la variable $i, \mu$ representa el promedio de las variables $i$ analizadas, y $\sigma$ corresponde a la desviación estándar de los promedios de las variables $i$. La media estandarizada alrededor del promedio, normaliza los valores entre $(-3)$ y $(+3)$, siendo $(0)$ el valor central que se corresponde con la media aritmética (Tabla 1).

Se obtuvo información de 66 docentes de postgrado, de los cuales 34 fueron recogidos en la Universidad "A" y 32en la Universidad "B". Para el análisis de las concepciones de inteligibilidad de la práctica docente, se acotaron las opciones alternativas de análisis (Monereo et al., 2000) sobre la base de principios como los de "Actividad y Participación; Motivación y Autoestima" (Stake, 2008). La codificación por palabras, frases y categorías semánticas, que dan sentido y significación al fenómeno estudiado (Checchia, 2009; Cook y Reichardt, 1997), fueron determinadas mediante frecuencias absolutas y estandarizadas de palabras significativas y frases mediante la aplicación del software de análisis de textos Nvivo 10.0.

\section{RESULTADOS}

Los resultados del estudio se presentarán, primero con una caracterización general, seguido de un análisis focalizado por Universidad para terminar con una caracterización de principios, categorías y cobertura.

\section{Caracterización general}

De los relatos obtenidos el año 2013 en la Universidad "A" y el año 2014 en la Universidad "B" (Tabla 1) se observa una interesante coincidencia que se expresa en el término "Actividades" utilizada en las dos universidades alcanzando los más altos puntajes con 2,35 y 1,50 puntos estándar respectivamente. Se pone así en evidencia que el foco docente gira coincidentemente alrededor de prácticas de formación de enseñanza basada en competencias, en este caso, ejercidas en el contexto de la formación profesional que vincula los objetivos profesionales de contexto con los objetivos pedagógicos de programas de postgrado.

En lo particular, los docentes de la Universidad "A", identifican "Actividades" con 2,35 puntos como conductores de su trabajo académico, "Conocimiento" con 1,65 y "Apoyar" con 1,30 puntos, evidenciando las prioridades de su modelo de conductas. Por otra parte, en posición opuesta, señalan términos como "Experiencias, Contexto y Crear" todas ubicadas en posición -1,16 a la izquierda de la media estandarizada. Por su parte los profesores de postgrado de la Universidad "B", mencionan "Competencias" con 1,94, 
"Actividades y Aprendizaje" con 1,50 puntos estándar, seguidas de "Conocimiento" con 1,43, y "Formación/formar" con 1,14 puntos estándar por encima de la media. Complementariamente identifican con menores frecuencias las conductas "Apoyar y Buscar" con -0,89 puntos, "Contexto" con -0,82 y "Responsabilidad" con - 0,75 puntos ubicadas a la izquierda de la media normal estándar.

En general, los docentes de postgrado de ambas universidades manifiestan conductas coincidentes cuando hablan de "Actividades" y "Conocimientos" difiriendo en los términos "Crear" y "Responsabilidad" pero orientadas en el mismo sentido de la formación de profesionales. Se observa, una evolución sistemática desde el "Enseñar conocimientos" hacia el "Desarrollo de competencias" profesionales, poniendo énfasis en la implementación de un plan institucional basado en la formación por competencias. Sin embargo, es interesante destacar la baja frecuencia de conceptos como "Creación y contexto" en tanto y en cuanto son términos que debieran fluir de manera explícita dentro del lenguaje que describen los docentes de sus actividades según la nueva modalidad de enseñanza por competencias.

Tabla 1: Frecuencia estandarizada $(\sigma)$ global por categoría de palabras entre rangos \pm 3

\begin{tabular}{|l|c|c|}
\hline \multirow{2}{*}{ Palabras } & \multicolumn{2}{|c|}{ Postgrado } \\
\cline { 2 - 3 } & $\begin{array}{c}\sigma: \\
\text { Universidad "A" 2013 }\end{array}$ & $\begin{array}{c}\sigma \\
\text { Universidad "B" 2014 }\end{array}$ \\
\hline Actividades & 2,35 & 1,50 \\
\hline Aprendizaje & 0,25 & 1,50 \\
\hline Desarrollar & 0,25 & 1,21 \\
\hline Conocimiento & 1,65 & 1,43 \\
\hline Contenidos & 0,60 & $-0,60$ \\
\hline Competencias & 0,60 & 1,94 \\
\hline Experiencias & $-1,16$ & $-0,67$ \\
\hline Manera & $-0,46$ & $-0,31$ \\
\hline Contexto & $-1,16$ & $-0,82$ \\
\hline Formación/formar & $-0,81$ & 1,14 \\
\hline Evaluación & $-0,11$ & $-0,53$ \\
\hline Responsabilidad & $-0,46$ & $-0,75$ \\
\hline Enseñar & 0,95 & $-0,31$ \\
\hline Buscar & $-0,81$ & $-0,89$ \\
\hline Metodologías/método & $-0,81$ & $-0,46$ \\
\hline Docencia & $-0,81$ & $-0,60$ \\
\hline Gestión & $-0,46$ & $-0,38$ \\
\hline Apoyar & 1,30 & $-0,89$ \\
\hline Implementación & 0,25 & $-0,75$ \\
\hline Crear & $-1,16$ & $-0,75$ \\
\hline
\end{tabular}

\section{Análisis focalizado por Universidad}

En la Universidad "A" (Tabla 2), se evidencia un perfil centrado en "Actividades y Profesional" con 1,95 puntos por encima de la media estándar seguida de "Conocimientos" con 1,27, mostrando las más altas frecuencias del enfoque basado en competencias. Por otra parte, con las frecuencias opuestas mencionan "Docencia, Gestión, Buscar y Metodología" todas con -1,11 puntos a la izquierda de la media estándar, significando los rezagos naturales del método de enseñanza tradicional por objetivos de aprendizaje.

De esta forma, en la Universidad "A", a partir de la implementación del método de enseñanza basada en competencias del año 2006 muestra conductas y prácticas que tienden a enriquecer la tarea del estudiante, conservando la posición jerárquica de los docentes en la entrega de conocimientos para el desarrollo de competencias profesionales.

En el caso de la Universidad "B", las frecuencias significativas se expresan en la "Docencia" con 2,93 puntos estandarizados y "Competencias" que logra 1,21 puntos por encima de la media, haciendo efectivo el tránsito desde el método de formación enseñanza - aprendizaje tradicional hacia el método basado en competencias. Sin perjuicio de lo señalado, las frecuencias más bajas se observan en los términos "Docencia" con $-1,12$, "Evaluación" con -1,05 y "Metodologías/Método" con -0,99 puntos por debajo de media estándar, demostrando la persistencia del método de enseñanza por objetivos que se ejerce en paralelo con las innovaciones del método basado en competencias. 
Tabla 2: Frecuencia estandarizada $(\sigma)$ por categorías docentes de postgrado

\begin{tabular}{|c|c|c|c|c|c|c|c|}
\hline \multicolumn{4}{|c|}{ Universidad "A" 2013} & \multicolumn{4}{c|}{ Universidad "B" 2014 } \\
\hline Palabras & $\sigma$ & Palabras & $\sigma$ & Palabras & $\sigma$ & Palabras & $\sigma$ \\
\hline Actividades & 1,95 & Desarrollar & $-0,09$ & Docencia & 2,93 & Educación & $-0,26$ \\
\hline Profesional & 1,95 & Evaluación & $-0,43$ & Competencias & 1,21 & Equipo & $-0,39$ \\
\hline Conocimiento & 1,27 & Manera & $-0,77$ & Actividad & 0,81 & Trabajar & $-0,39$ \\
\hline Apoyo & 0,93 & Responsabilidad & $-0,77$ & Aprendizaje & 0,81 & Control & $-0,59$ \\
\hline Enseñanza & 0,93 & Gestión & $-0,77$ & Conocimiento & 0,74 & Manera & $-0,85$ \\
\hline Competencias & 0,25 & Docencia & $-1,11$ & Desarrollo & 0,54 & Enseñar & $-0,85$ \\
\hline Contenidos & 0,25 & Buscar & $-1,11$ & Formación & 0,48 & Gestión & $-0,92$ \\
\hline Aprendizaje & $-0,09$ & Formación & $-1,11$ & Labor & 0,34 & Metodología/Método & $-0,99$ \\
\hline Pedagógica & $-0,09$ & Metodología & $-1,11$ & Proceso & $-0,19$ & Evaluación & $-1,05$ \\
\hline Implementación & $-0,09$ & & & Práctica & $-0,26$ & Docencia & $-1,12$ \\
\hline
\end{tabular}

\section{Caracterización de principios, categorías y cobertura}

En general, los principios orientadores de la actividad docente en las dos universidades (Tabla 3 ) sigue el mismo patrón de conducta, con docentes guiados por las categorías "Globalización" y "Personalización" alcanzando 1,62 puntos estándar y, en posición opuesta las categorías "Motivación y Autoestima", además de "Aprendizajes Significativos" con -0,54 puntos a la izquierda de la distribución normal.

Tabla 3: Frecuencia estandarizada $(\sigma)$ de principios orientadores y cobertura $(\%)$ de palabras a nivel de postgrado

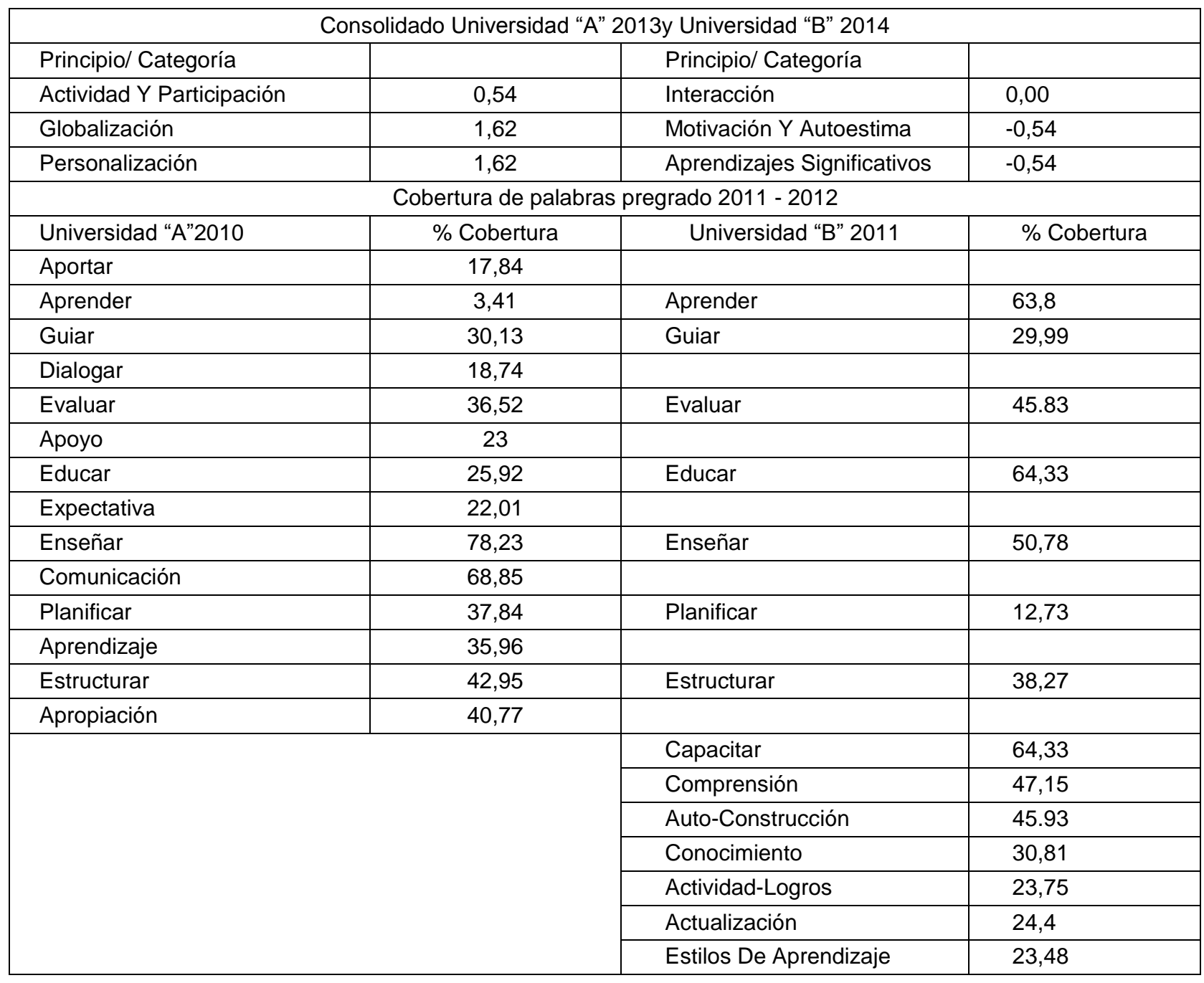


Los profesores de la Universidad "A" determinan como relevantes las categorías "Enseñar" alcanzando un $78,23 \%$ de cobertura, seguido de la categoría "Comunicación" con $68,85 \%$ de frecuencia relativa lo que infiere una contradicción evidente entre el enfoque de enseñanza basado en competencias, que parece bastante bien instalado en la conducta de los profesores, pero que sin embargo, parece no estar lo suficientemente arraigado a nivel de postgrado. Las categorías mencionadas explicitan la importancia que se asigna a las actividades de transmisión de contenidos a la usanza tradicional, de formación por objetivos de aprendizaje. Con un peso relativo medio se menciona la categoría "Estructurar" con $42,95 \%$ de cobertura respecto de las actividades en aula, dando cabida a la utilización de métodos de enseñanza-aprendizaje contextualizados. Los docentes exponen así mismo la relevancia de la actividad de "Apropiación" con un 40,77\% de cobertura validando el rol activo que le cabe al estudiante en su propio aprendizaje.

Sin perjuicio de lo señalado, la categoría "Aprender" con tan sólo un 3,41\% de cobertura remite al principio de "aprendizajes significativos" y, a través de ello, a las actividades relacionadas con los principios "Actividad y Participación, Globalización, Personalización, e Interacción" de la formación profesional donde el aprendizaje es el resultado de la categoría "Aportar" (17,84\% de cobertura) a través de la cual el docente trabaja de manera personalizada y singular asumiendo el hecho que no todas las personas aprenden de la misma manera y su rol es actuar en complementariedad.

A nivel de la Universidad "B", se ponen en evidencia concepciones docentes arraigadas cuando los docentes expresan las categorías "Educar" y "Capacitar", ambas con un registro relevante de 64,33\% de cobertura, situando a los profesores en el modelo de enseñanza por objetivo que se complementa con los términos "Educar" y al mismo tiempo, los ubica en el modelo de enseñanza basada en competencias cuando priorizan las acciones de "Capacitar". Seguidamente y muy cerca de esta misma dicotomía se encuentra la categoría "Aprender" con un 63,8\% de cobertura con la palabra "Enseñar" que alcanza un 50,78\%, en ambos casos derivadas del modelo tradicional de enseñanza y que se extiende en ese mismo sentido a través de la categoría "Comprensión" con un 47,15\% de cobertura.

Finalmente, con un discreto $45,93 \%$ de cobertura la categoría "Auto-Construcción" del conocimiento, abre la puerta a la enseñanza basada en competencias, pero mantiene elementos de la enseñanza tradicional cuando el ciclo de enseñanza-aprendizaje se cierra con las actividades derivadas de la categoría "Evaluar" que alcanza un destacado $45.83 \%$ de cobertura. Por último, entre las categorías de actividades menos mencionadas se destaca "Planificar" con un $12,73 \%$ de cobertura que, aunque proviene del modelo tradicional de enseñanza por objetivos, está comenzando a mutar hacia la enseñanza basada en competencias incorporando diversas y variadas formas de autoevaluación, co-evaluación y hetero- evaluación entre los métodos aplicables a nivel de postgrado.

\section{CONCLUSIONES}

Del análisis de resultados, su discusión y de los antecedentes expuestos, se pueden obtener las siguientes conclusiones principales:

1. En general, se determina una dicotomía entre declaración y práctica docente que se enfoca más en el modelo de enseñanza cognitiva que en el desarrollo de capacidades y competencias.

2. Los principios orientadores en la Universidad "A" enfatizan el enseñar y comunicar del docente, en tanto que en la Universidad "B", se focaliza el capacitar del docente junto al aprender del alumno.

3. En la Universidad "A" se observa un perfil docente enfocado lo pedagógico - cognitivo centrado en el esfuerzo del docente que se responsabiliza de enseñar; en tanto que en la Universidad "B" se evidencian concepciones ambivalentes que los sitúan, entre la enseñanza de cargo del docente y el desarrollo de competencias, de responsabilidad del estudiante.

4. En general, la valoración de la enseñanza basada en competencias del profesorado es positiva, por la novedosa manera de aprender que se promueva en los alumnos, las habilidades trabajo en grupo y destreza que deben poner en actuación y por el efecto que provoca en los estudiantes el nivel de compromiso y responsabilidad que asumen, puesto que los hace partícipes del proceso de adquisición de conocimientos y destrezas que conforman las competencias profesionales buscadas.

\section{REFERENCIAS}

Abela, D. A., Las Técnicas de Análisis de Contenido: Una Revisión Actualizada (en línea) Documentos de Trabajo, Fundación Centro de Estudios Andaluces, Sevilla, Serie Sociología (2002) http://books.google.cl/ Acceso: 20 de mayo (2013) 
Barbier, J.M., Prácticas de Formación: Evaluación y Análisis. 1a edición, Editorial Novedades Educativas, Buenos Aires, Argentina (1999)

Barbier, J.M., Valeurs et Activités Professionnelles Séminaire du Centre de Recherche Sur la Formation. 1a édition, Éditions L'Harmattan, Paris, France (2004)

Barbier, J.M. y O. Galatanu, L'analyse de la Singularité des Actions: Quelques Outils Conceptuels, En CRFCNAM L'analyse de la singularité de l'action, pp 13-51, Presses Universitaires de France, Paris, France (2000)

Bustamante, M., C. Cisternas y P. Villarreal, Evolución de Competencias Profesionales en el Sector de la Salud, Chile, http://www.educandus.cl/ojs/index.php/fcompetencias/article/viewFile/57/56; ISSN: ISSN 07188757, Revista Electrónica de Desarrollo de Competencias (REDEC), 2(4), 105-117 (2009)

Camargo, I.M. y C. Pardo, Competencias Docentes de Profesores de Pregrado: Diseño y Validación de un Instrumento de Evaluación, http://www.redalyc.org/articulo.oa?id=64770211; ISSN: 1657-9267, Univ. Psychol., 7(2), 441-455 (2008)

Cardoso, E.O., Cerecedo, M.T. y Vanegas, E.A., Las Competencias Docentes en los Programas de Posgrado en Administración: Un Estudio de Diagnóstico, doi: 10.4067/S0718-50062013000200006, Form. Univ., 6(2), 43-50 (2013)

Castaño, E.M. y Garín, A., Incorporación de Personas Adultas a los Estudios Universitarios: Aprendizaje Permanente para Arquitectos Técnicos, doi: 10.4067/S0718-50062012000300003, Form. Univ., 5(3), 17-26 (2012)

Checchia, B., Las Competencias del Docente Universitario (en línea) (2009), http://www.fvet.uba.ar/institucional/subir/adjuntos/userfiles/COMPETENCIASDOCENTES.pdf. Acceso: 10 de Marzo (2013)

Cook, T., y C. Reichardt, Métodos Cualitativos y Cuantitativos en Investigación Evaluativa. 1aㅡ edición, Ediciones Morata, Madrid, España (1997)

Davini, M.C., Métodos de Enseñanza. Didáctica General para Maestros y Profesores. $1^{\text {a }}$ edición, Editorial Santillana, Buenos Aires, Argentina (2008)

De Miguel, M., Modalidades de Enseñanza Centradas en el Desarrollo de Competencias: Orientaciones para Promover el Cambio Metodológico en el Espacio Europeo de Educación Superior (en línea), 1a edición, Oviedo, España, (2005), http://www.uvic.es/sites/default/files/Ensenanza_para_competencias.PDF. Acceso: 10 de Marzo (2013)

Díaz, C., Martínez, P., Roa, I., y Sanhueza, M.G., Los docentes en la sociedad actual sus creencias y cogniciones pedagógicas respecto al proceso didáctico, doi: 10.4067/S0718-65682010000100025, Polis, 9(25), 421-436 (2010)

Didou, S., Educación Superior, Identidad Cultural y Globalización: las Experiencias Europeas en la Perspectiva Mexicana, http://148.206.53.230/revistasuam/iztapalapa/include/getdoc.php?id=512\&article=520\&mode=pdf; ISSN 0185-4259, IZTALAPAPA, Revista de Ciencias Sociales y Humanidades, 38(especial), 191-202 (1996)

Gagné, R., Las Condiciones del Aprendizaje, Editorial Aguilar, Madrid, España (1971)

García, B., J. Loredo y G. Carranza, Análisis de la Práctica de los Docentes: Pensamiento, Interacción y Reflexión, http://redie.uabc.mx/index.php/redie/article/view/200; ISSN: 1607-4041, Revista Electrónica de Investigación Educativa, Especial, 1-15 (2008)

Glaser, B. y A. Strauss, The Discovery of Grounded Theory: Strategies for Qualitative Research, Aldine Transaction, New York, USA (1999)

Jiménez, M., Reveco, J., González, M. y Astudillo, C., Dilemas Ideológicos ante una Formación Universitaria con Sello Valórico Distintivo. El Caso de una Carrera de Ingeniería, doi: 10.4067/S0718-50062013000400003, Form. Univ., 6(4), 13-26 (2013)

Krippendorff, K., Metodología de Análisis de Contenido. Teoría y Práctica, Ediciones Paidós, Barcelona, España (1990) 
Litwin, E., El Oficio de Enseñar, Editorial Paidos, Buenos Aires, Argentina (2008)

Marín-García, J.A. y Conchado-Peiró, A., Influencia de los Perfiles Docentes en el Uso y Preferencia de Dinámicas de Trabajo en Grupo con Alumnos Universitarios, doi: 10.4067/S0718-50062012000500002, Form. Univ., 5(5), 3-14 (2012)

Monereo, C. (coord.), M. Castelló, M. Clariana, M. Palma y M.L. Pérez, Estrategias de Enseñanza y Aprendizaje Formación del Profesorado y Aplicación en la Escuela, 8ª edición, Editorial Graó, México D.F., México (2000)

Moreno, M.P., Nava, M. y Campos, M., Los Comentarios Abiertos como Referente de Evaluación de la Docencia Universitaria: La Conveniencia de su Interpretación y Tratamiento, doi: 10.4067/S071850062014000100006, Form. Univ., 7(1), 41-48 (2014)

Muñoz, F., La Necesidad de Regular la Docencia Universitaria en Chile: una Propuesta de Lege Ferenda, doi: 10.4067/S0718-34372012000300019, Rev. Chil. Derecho, 39(3), 891-907 (2012)

Palominos, F., Mendez, M. y Barrera, R., Sistema de Perfeccionamiento Orientado a Competencias para Docentes de la Educación Superior, doi: 10.4067/S0718-50062014000300003, Form. Univ., 7(3), 1-22 (2014)

Patiño, L. y M. Rojas, Subjetividad y Subjetivación de las Prácticas Pedagógicas en la Universidad. Educ. Educ., 12(1), 93-105 (2009)

Pedraja, L., Desafíos para el Profesorado en la Sociedad del Conocimiento, http://www.scielo.cl/pdf/ingeniare/v20n1/art14.pdf; ISSN 0718-3305, Ingeniare. Rev. Chil. Ing., 20(1), 136-144 (2012)

Perrenoud, P., La Formación de los Docentes en el Siglo XXI (en línea)http://www.unige.ch/fapse/SSE/teachers/perrenoud/php_main/php_2001/2001_36.html(2001) Acceso: 10 de Marzo (2013)

Rodríguez, M., Mena, D. y Rubio, C., Competencias que pueden Desarrollarse en la Asignatura de Mecánica en el Currículo de Ingeniería, doi: 10.4067/S0718-50062011000400002, Form. Univ., 4(4), 3-12 (2011)

Ruiz, J., Análisis Sociológico del Discurso: Métodos y Lógicas, http://www.qualitativeresearch.net/index.php/fqs/article/view/1298/2776; ISSN 1438-5627, Forum: Qualitative social research, 10(2), Art. 26 (2009)

Siqueira, I., Inclusion and Solidarity as Education Methodology, doi: 10.5901/jesr.2012.v2n7p75; ISSN: 2239978X, Journal of Educational and Social Research, 2(7), 75-81 (2012)

Stake, R., La Ventaja de los Criterios, la Esencialidad del Juicio, http://www.rinace.net/riee/numeros/vol1num3_e/art2.pdf; ISSN 1989-0397, Revista Iberoamericana de Evaluación en Educación, 1(3e), 18-28 (2008)

Zabalza, M.A., Competencias Docentes del Profesorado Universitario: Calidad y Desarrollo Profesional, $2^{\mathrm{a}}$ edición, Editorial Narcea. S.A. de ediciones, Madrid, España, (2007) 